\title{
Tensor Permeability Measurements at L-Band Frequencies Using a Degenerate Mode Cavity
}

\author{
L. B. Schmidt, * R. D. Harrington, ${ }^{*}$ and W. E. Case * \\ Institute for Basic Standards, National Bureau of Standards, Boulder, Colo. 80302
}

(October 18, 1966)

\begin{abstract}
The exact solution for the field equations of a cylindrical $\mathrm{TM}_{110}$ mode cavity has previously allowed accurate measurements of tensor permeability to be obtained at X-band frequencies. It is demonstrated that this method is also applicable at frequencies down to $1 \mathrm{GHz}$. A brief description of the cavity and measurement system for obtaining data at these lower frequencies is given. Both intrinsic and external permeability results on three commercially available polycrystalline garnets are shown. The larger size rods required for measurements at these frequencies result in some sample size effects in the data. In addition, a previously unreported absorption in the external tensor permeability of the materials was observed.
\end{abstract}

Key Words: Degenerate mode cavity, L-band cavity, polycrystalline garnets, tensor permeability.

\section{Introduction}

A knowledge of the tensor permeability components as described by Polder [1] ${ }^{1}$ of a ferrimagnetic material is often required in the theory and design of ferrite devices at microwave frequencies. Considerable effort has been directed towards the measurement of these parameters in recent years. Most of these tensor permeability studies have been carried out using cavity techniques [2-18]. In particular, degenerate mode cavities excited with either a positive or negative pure circularly polarized wave have been the most accurate means available for obtaining measurements of this type. Both TE and TM mode cavities have been used in this manner. In comparing the degenerate mode cavity methods, the measurement of rod-shaped samples placed along the axis of a $\mathrm{TM}_{110}$ mode cylindrical cavity has been particularly attractive since this geometry allows an exact solution to be obtained for the field equations [19-20].

The measurement of tensor permeability using the $\mathrm{TM}_{110}$ mode cavity as well as any of the other cavity methods has in general been confined to frequencies of $3 \mathrm{GHz}$ or higher. Exceptions to this may be found in the work of Ogasawara [21-23] as well as Waldron and Maxwell [24-27], who use stripline techniques for measurements from $3 \mathrm{GHz}$ down to the UHF range. Ogasawara describes measurements of disks in crossed stripline cavities. Waldron and Maxwell use thin rectangular plates in a single stripline cavity. In

\footnotetext{
*Radio and Microwave Materials, Radio Standards Physics, NBS Laboratories, Boulder Colo. 80302

${ }^{1}$ Figures in brackets indicate the literature references at the end of this paper.
}

either case, the use of disk or rectangular shaped samples in stripline may be subject to errors due to sample geometry effects, nonuniform electromagnetic fields, etc. [28-29].

It appears feasible that some of the errors associated with sample measurements in stripline could be avoided, at least in the frequency range down to about $1 \mathrm{GHz}$, by the use of the previously mentioned $\mathrm{TM}_{110}$ mode cylindrical cavity at these lower frequencies. This cavity offers advantages over other waveguide cavities at lower frequencies for two reasons. First, this mode allows a flat cylindrical type cavity to be designed which is sufficiently thin along the axis, even at these lower microwave frequencies, to fit between the pole pieces of an electromagnet. Second, the rod-shaped samples and exact solution for the field equations mentioned above should help to avoid sample shape errors appearing in other methods.

This paper describes a feasibility study which uses a $\mathrm{TM}_{110}$ mode cavity for tensor permeability measurements in the L-band region. A brief description is given of the measurement method, the cavity, and the associated circuitry used in these measurements. Several improvements in the $\mathrm{TM}_{110}$ mode cavity are noted which are also applicable to the use of this method at higher frequencies. This is followed by a description of typical data obtained on three commercially available polycrystalline garnets at $1106 \mathrm{MHz}$.

\section{Measurement Technique}

\subsection{General Method}

The general procedure for measuring tensor permeability using degenerate mode cavities is based on 
the fact that the magnetic flux $b$ in a magnetic material is related to a circularly polarized field $h_{i}$ inside the material by the relation [4]

$$
b_{ \pm}=(\mu \pm \alpha) h_{i_{ \pm}} .
$$

In this equation, $\mu$ and $\alpha$ are both complex and are the diagonal and off diagonal components of the intrinsic tensor permeability. The subscripts refer to either a positive or a negative circularly polarized wave.

In a typical measurement, the frequency shift and change in $Q$ of a cavity containing a small sample are determined as a function of an applied d-c field for each of the two senses of circular polarization. In most cases, the experimental relationship between $(\mu \pm \alpha)$ and the changes in the resonant frequency and $Q$ of the cavity are determined from cavity perturbation theory. However, in the case of the $\mathrm{TM}_{110}$ mode cylindrical cavity containing a rod-shaped sample along the axis, the correspondingly more exact relations may be obtained from a direct application of Maxwell's equations to the sample and cavity geometry as shown by Bussey and Steinert [19-20]. This approach is well described in their papers and will not be repeated here. It suffices to point out that the intrinsic permeability data in this paper have been calculated with the aid of a computer from eqs (10) and (11) of their paper $[20] .^{2}$

To accurately identify the resonant field and the maximum value of the imaginary part of $(\mu+\alpha)$, it was necessary to know the d-c field value at increments of one oersted immediately above and below resonance. The accuracy of the available equipment for measuring the d-c field in the range of values used in these measurements was not adequate to obtain this resolution. The relation between the measured frequency and the applied d-c field was very nearly linear. This made it possible to plot this relation and interpolate between experimentally observed points. The experimental data and the data obtained by this interpolation were combined to obtain the intrinsic permeability data shown in this paper.

It should be emphasized that the permeability $(\mu \pm \alpha)$ defined in eq (1) and calculated from the formulas of Bussey and Steinert is the intrinsic permeability which does not depend upon the geometry of the sample. This situation arises because the field $h_{i}$ in eq (1) refers to the rf field inside the sample. In some cases a so-called "external" permeability $(\eta \pm \kappa)$ has been referred to in the literature. This is defined in terms of the rf field $h_{0}$ existing in the absence of the specimen. In this case, we have an analogy to eq (1)

$$
b_{ \pm}=(\eta \pm \kappa) h_{0_{ \pm}},
$$

where $\eta$ and $\kappa$ are again complex and are the diagonal and off diagonal components of the external permea-

${ }^{2}$ The signs preceding the $\epsilon^{\prime \prime}$ and $\epsilon^{\prime}$ terms of eq (11) in ref. 20 should be - and + respectively rather than as given in the paper. This is because the expression for inverse permeability $m^{*}$ should have been $m^{*}=m^{\prime}-i m^{\prime \prime}$ rather than $m^{\prime}+i m^{\prime \prime}$ which the authors used in their discussion. bility. In the case of a rod sample, Waldron [30] has shown that the external permeability is related to intrinsic permeability by the relation

$$
\eta \pm \kappa=\frac{2(\mu \pm \alpha)}{1+(\mu \pm \alpha)}
$$

In this paper, we are primarily concerned with the evaluation of the intrinsic permeability. However, the above concept of external permeability is included primarily because we have observed previously unreported absorption phenomena.

\subsection{Cavity Details}

A simplified drawing of the circular $\mathrm{TM}_{110}$ mode cavity developed for making tensor permeability measurements at L-band frequencies is shown in figure 1. A resonant frequency of $1106 \mathrm{MHz}$ was chosen which results in a cavity having an inside diameter of about 13 in. and an inside height along the axis of approximately 1.4 in. As previously mentioned, this readily allows the cavity to be placed between the pole pieces of a typical electromagnet since the magnetic field is applied parallel to the axis of the sample and cavity.
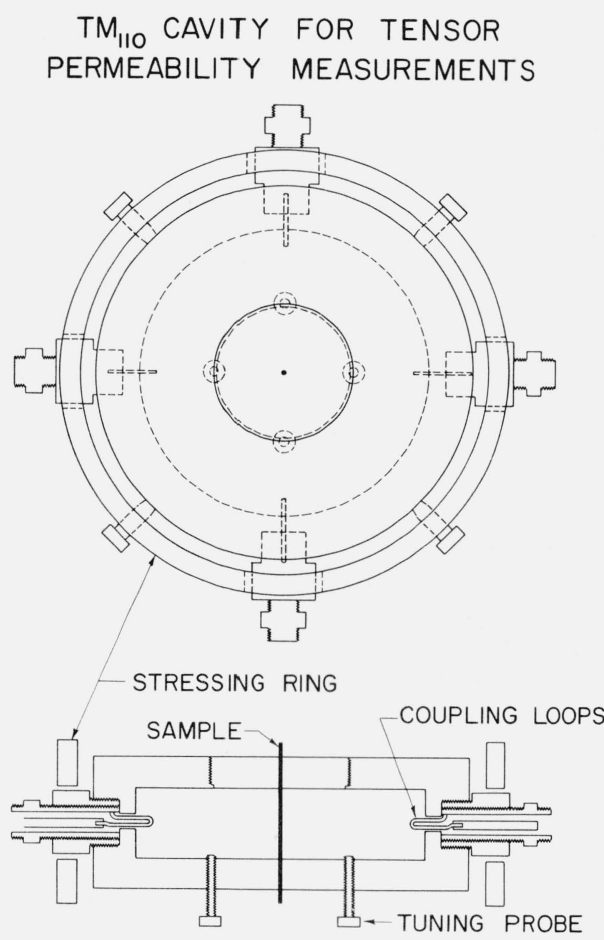

FIGURE 1. Simplified drawing of $T M_{110}$ mode cavity for tensor permeability measurements at $1106 \mathrm{MHz}$. 
The cavity itself was fabricated from a solid piece of brass with an access lid having a radius of 48 percent of the inside radius of the cavity which corresponds to a low current region. The solid piece of brass was employed to avoid the use of mechanical joints in the regions of high current such as at the periphery of the cavity. Earlier designs of cylindrical cavities using lids that made contact at the corners demonstrated that it is difficult to obtain a perfectly symmetrical contact resistance around the periphery of the cavity. Tuning probes are located in the region of high electric field on the solid end of the cavity as seen in figure 1 . It was observed that better circular polarization conditions could be obtained by using the above techniques.

Further minor adjustments are still necessary for obtaining optimum circular polarization conditions in the cavity. For example, as shown in figure 1, a heavy ring clamp is placed around the outer circumference of the cavity with adjusting screws for stressing the cavity in compression. This allows slight adjustments to be made in the circular symmetry of the cavity. Four sinall tuning probes as shown in figure 1 were placed in line with the coupling loops and at the point of maximum electric field to provide further fine adjustments in the tuning of the cavity. Adjustable coaxial coupling loops were also used. These of course readily allow coaxial lines, rather than waveguide, to be used external to the cavity which is advantageous at these lower frequencies. At the same time, the coaxial loops easily allow small adjustments in the coupling to be made in order to obtain a pure circularly polarized mode.

The procedure for obtaining circularly polarized waves in the cavity is carried out by first adjusting the compression screws, tuning probes, and coupling loops of the empty cavity in an iterating procedure to equalize the magnitude and frequency of the two possible modes of the degenerate mode cavity. Further adjustments are then made by placing a ferrite rod in the cavity and observing any resulting mode splitting when the ferrite is biased with a d-c field. Final adjustments using the coupling loops and line stretchers, which are external to the cavity, are then made in an effort to completely suppress one mode when the cavity is excited by a circularly polarized wave. It should be noted that it is more difficult to obtain the final adjustments with the sample in the cavity at L-band frequencies than at the higher frequencies. This is partly because the greater low field losses make it more difficult to distinctly see the splitting of the two modes. However, as will be shown in this paper, it is still possible to obtain good data.

Figure 2 is a photograph of the cavity showing many of the features described above. A typical sample is shown at the center of the cavity. In an actual measurement, the sample would be inserted through the small hole in the lid and cavity as shown. The lid would of course be on the cavity and would not be opened during the measurement. The small rod protruding from the stressing ring in the lower righthand corner of the photo is used for hanging the cavity between the pole pieces of the electromagnet.

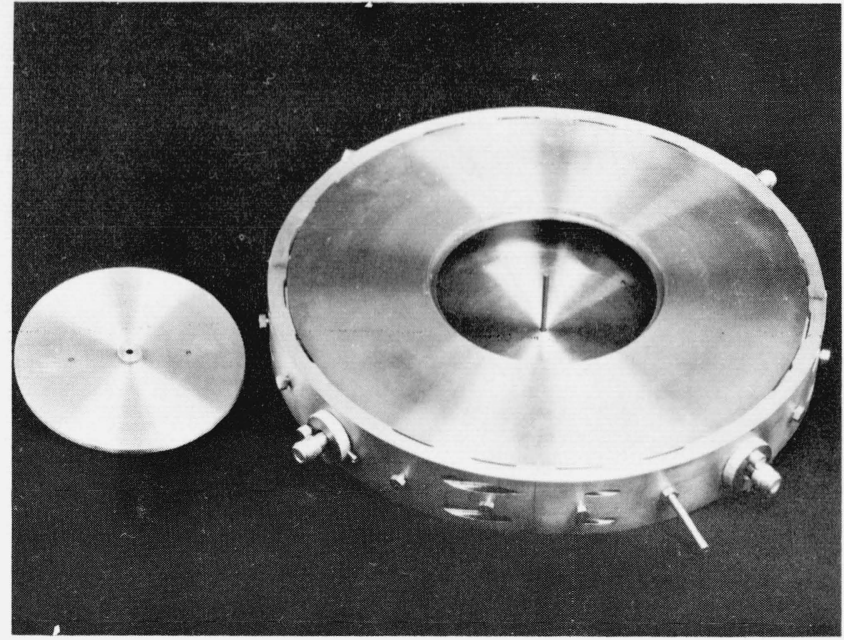

FIGURE 2. Photograph of $T M_{110}$ mode cavity with lid removed showing typical sample.

\subsection{Measurement System}

The basic requirements in the circuit for tensor permeability measurements are shown in figure 3 . The equipment used in this system is derived from commercially available coaxial components modified where necessary to meet the needs of these measurements. In particular, the microwave source is a commercial oscillator whose power supply was replaced by well-regulated direct current power supplies to improve the quality and stability of the signal. The plate supply was regulated to 0.002 percent, and the filament supply regulation was adequate at 0.5 percent. The system also makes use of IF detection to increase sensitivity and a phase-locked local oscillator system was used to assist in tracking the microwave source during the measurements [31].

Optimum accuracy in the resonant frequency and $Q$ measurements of the cavity was assured by using a transfer oscillator in conjunction with a frequency counter. The crystal detector and galvanometer was in turn calibrated using a $30-\mathrm{MHz}$ piston attenuator in the IF circuit in order to improve the accuracy of the 3 - $\mathrm{dB}$ points on the cavity resonance curve used in the $Q$ determinations.

The equal $90^{\circ}$ phase shifted signals needed for circular polarization are obtained by the phase and power splitter in conjunction with the line stretchers. These adjustable length coaxial lines readily allow fine adjustments to be made on the phase which is necessary for obtaining good circular polarization.

\section{Measurement Results}

The permeabilities of three commercially available polycrystalline garnet materials of different magnetizations were measured at $1106 \mathrm{MHz}$. These materials will be identified as follows: 


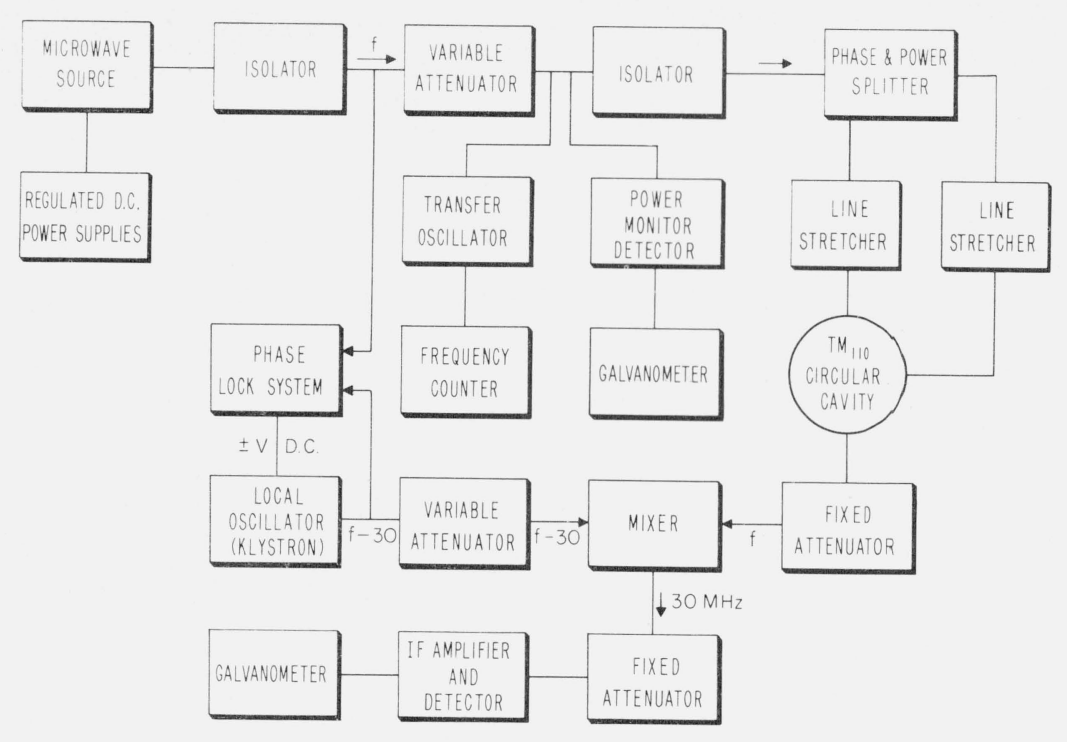

FIGURE 3. Schematic diagram of circuit components used in tensor permeability measurements at L-band frequencies.

\begin{tabular}{c|c|c}
\hline \hline Material & Composition & $\begin{array}{c}\text { Saturation magnetization, } \\
4 \pi \mathrm{M} \text { in gauss }\end{array}$ \\
\hline & Substituted YIG & 580 \\
A & Substituted YIG & 1158 \\
C & YIG & 1799 \\
\hline
\end{tabular}

Measurements were made on rods of diameters $0.100,0.150,0.200,0.250$, and 0.300 in. Although data could be obtained on all these rods, the smaller diameters $(0.1$ in -0.15 in) resulted in poor precision because of the resulting small frequency shifts while the large rods $(0.3 \mathrm{in})$ proved difficult to measure because of the greater loss introduced into the system. The best data were obtained on the 0.250 in rods.

The real and imaginary parts of the intrinsic tensor permeability values, $\left(\mu^{\prime} \pm \alpha^{\prime}\right)$ and $\left(\mu^{\prime \prime} \pm \alpha^{\prime \prime}\right)$, for the above three materials using 0.250 in diameter rods whose lengths ranged from 2.850 in to 2.882 in are shown in the top graph of figures 4,5 , and 6 . It is of interest that these relatively good tensor permeability resonance curves were obtained on rod samples whose ordinary Kittel resonance properties could not be measured at L-band frequencies using a rod geometry due to the low fields resulting in unsaturated conditions in the sample.

It is feasible that low field effects may also be influencing the accuracy of these tensor permeability measurements. For example, we have shown the external tensor permeability for positive circular polari-

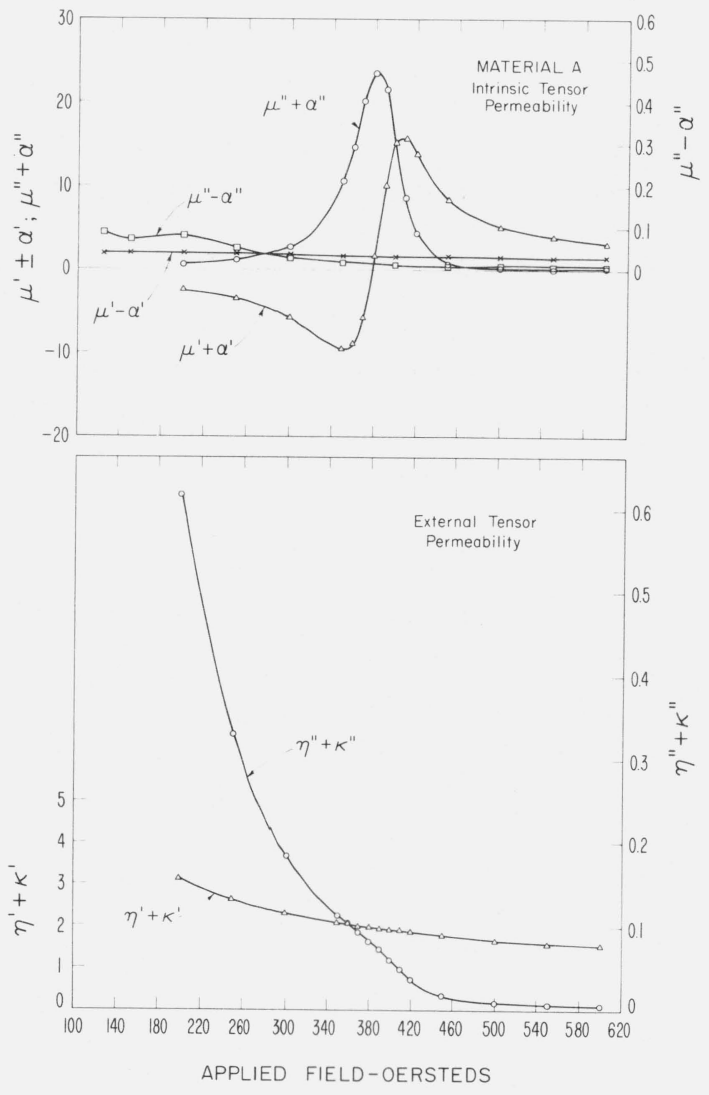

FIGURE 4. Intrinsic and external tensor permeabilities for polycrystalline substituted yttrium iron garnet, material $A, 4 \pi \mathrm{M}=$ $580 \mathrm{G}$. 


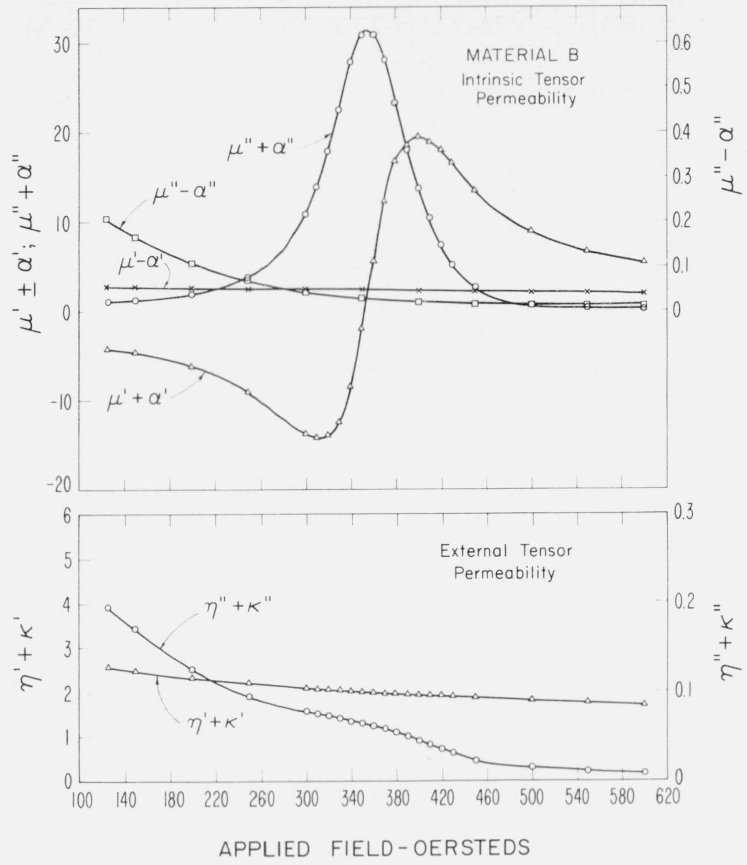

Figure 5. Intrinsic and external tensor permeabilities for polycrystalline substituted yttrium iron garnet, material $B, 4 \pi \mathrm{M}=1158 \mathrm{G}$.

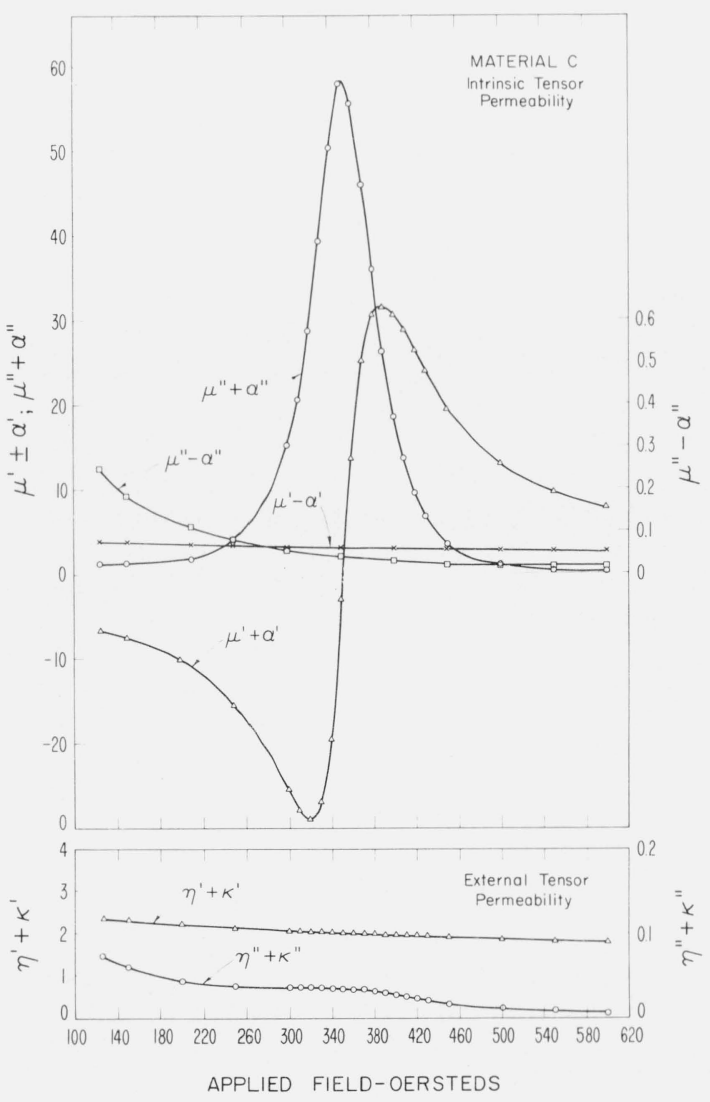

FIGURE 6. Intrinsic and external tensor permeabilities for polycrystalline yttrium iron garnet, material $C, 4 \pi \mathrm{M}=1799 \mathrm{G}$. zation conditions corresponding to the above intrinsic tensor results in the bottom graphs of figures 4,5 , and 6. These curves were calculated from the intrinsic tensor values using eq (3). The external permeability curves are essentially just the high field side of the Kittel resonance curve. Based on data obtained at higher frequencies [5], both $\eta^{\prime}+\kappa^{\prime}$ and $\eta^{\prime \prime}+\kappa^{\prime \prime}$ might be expected to be smoothly decreasing curves. However, in all three cases, a slight absorption was observed in the $\eta^{\prime \prime}+\kappa^{\prime \prime}$ data in the vicinity of the intrinsic tensor permeability resonance. This same phenomena was similarly observed in measuring one of the rods in a rectangular $\mathrm{TE}_{102}$ rectangular cavity at $1107 \mathrm{MHz}$ indicating that it probably is not related to the adjustment of the circular polarization in our cylindrical cavity. It was also observed on rods ground into prolate ellipsoids indicating that it is not a macroscopic geometry effect. It is probably related to the relatively low fields used in these experiments although further studies are necessary in this area.

It is also of interest that the small absorption located at a field of about 200 Oe in the $\mu^{\prime \prime}-\alpha^{\prime \prime}$ curve for material A in figure 4 appeared in measurements of different size rods of the same material and also in a prolate ellipsoid formed from one of the rods. This phenomena again may be characteristic of this material at low fields.

Further studies were carried out in an effort to determine the precision and accuracy of the system for measuring the intrinsic tensor permeability which was of primary interest in our program. First of all, it was observed that, for any given rod, the field for maximum absorption in $\mu^{\prime \prime}+\alpha^{\prime \prime}$ could be repeated to better than 1 percent while the linewidth and maximum value of $\mu^{\prime \prime}+\alpha^{\prime \prime}$ at resonance could be repeated to better than 5 percent. However, it was noted that data obtained on different rods may show a much greater variation than this which is probably due to sample inhomogeneity effects, etc.

The linewidths of the three materials obtained from the plotted results in figures, 4,5 , and 6 were compared with corresponding measurements on disk samples in a rectangular $\mathrm{TE}_{102}$ mode cavity at $1107 \mathrm{MHz}$ using the technique adopted by the ASTM [32]. Disks having aspect ratios (diameter/thickness) of the order of 83 to 85 were used in an effort to minimize shape effects [33]. The results are shown in the following table:

\begin{tabular}{c|c|c}
\hline \hline \multirow{2}{*}{ Sample } & \multicolumn{2}{|c}{ Linewidth (oersteds) } \\
\cline { 2 - 3 } & $\begin{array}{c}\text { Tensor meas. } \\
\text { (rods) }\end{array}$ & $\begin{array}{c}\text { ASTM meas. } \\
\text { (disks) }\end{array}$ \\
\hline & 51 & 48 \\
A & 82 & 78 \\
C & 66 & 62 \\
\hline
\end{tabular}


The agreement is remarkably good considering the variations that are often experienced even from samples cut from the same slab of material. These results indicate that the tensor results give a very good indication of the linewidth in addition to providing information on the individual tensor permeability components.

It is also recognized that the rods used at these frequencies do not approximate infinitely long cylinders, and demagnetizing corrections may be necessary. For example, it is well known that the internal field $H_{i}$ in a material is related to the external field $H_{0}$ by the relation

$$
H_{i}=H_{0}-N M
$$

where $N$ is the demagnetizing factor and $M$ is the magnetization. The data shown in figure 7 for the three materials indicate that the applied field for resonance, $H_{o}$, does increase slightly with increasing rod diameter. Better results are obtained using the corresponding data for the internal field $H_{i}$ calculated using eq (4), and $N$ values obtained from Bozorth [34], with values of $M$ corresponding to the internal field as determined independently with a magnetometer. It thus appears that small size effects in resonant field values can be expected; however, these can be partially corrected for in the case of the rod samples.

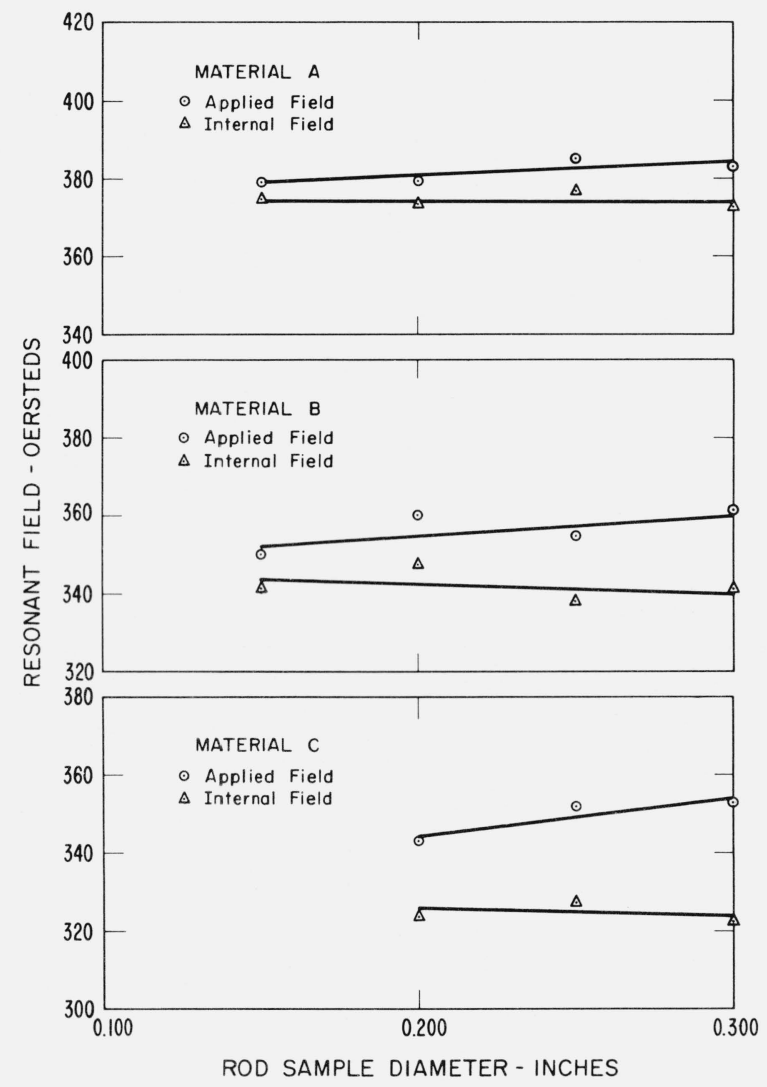

FIGURE 7. Dependence of intrinsic tensor permeability resonant field on sample diameter for materials $A, B$, and $C$.
No definite trend in linewidth on rod diameter could be identified within the variations of data observed on different rods. The linewidth varied considerably on different size rods that were cut from the same slab; therefore, it is possible that some size effects may still be present within the total variation.

It is apparent that some of the above variations in resonant field and linewidth could be comparable to similar variations on size previously observed in ferromagnetic resonance studies on disks [33]. It is also of interest to note that differences in the properties of ellipsoids and rods using the $\mathrm{TM}_{110}$ cavity, as well as ellipsoids and disks measured in the previous program, were observed. It thus appears that rod samples in the $\mathrm{TM}_{110}$ cavity as well as thin disk samples such as might be used in a stripline cavity may both result in some sample-shape errors in tensor permeability measurements.

Although the $T M_{110}$ cavity method does not completely eliminate the problem of sample shape errors occurring in other methods, the cylindrical symmetry and ability to make several adjustments on the cavity for obtaining circular polarization may have advantages over stripline methods using disks. Furthermore, the cylindrical cavity allows the use of a long rod sample which is a distinct advantage when data in the vicinity of the intrinsic tensor permeability resonance are desired since this resonance coincides with the ordinary ferromagnetic resonance in disks but not in rods. The complications introduced by this higher loss in the disks have been previously recognized at higher frequencies [6] and further substantiated by our own efforts to measure disks at these lower frequencies.

\section{Conclusions}

On the basis of the above study, it is concluded that a degenerate $\mathrm{TM}_{110}$ mode cavity is very satisfactory for measuring the tensor permeability of rod samples at frequencies as low as $1 \mathrm{GHz}$. It is possible to obtain good linewidth measurements on cylindrical samples which ordinarily could not be measured at these frequencies using standard rectangular waveguide techniques normally used in studies of the Kittel resonance.

Although the use of rods in this method offers some decided advantages over disks in stripline when measuring intrinsic tensor permeability near resonance, the $\mathrm{TM}_{110}$ cavity method is still influenced by some sample shape effects. It is possible that all low frequency tensor permeability measurements may be influenced by sample shape effects that are related to the low fields involved. These results along with the appearance of a small anomalous absorption in the external permeability observed in our rod data in the vicinity of the intrinsic tensor permeability resonance indicate that there is still a need for further work describing ferromagnetic resonance and tensor permeability properties at low frequencies and applied fields. 
The authors express their appreciation to H. E. Bussey for helpful discussions regarding the $\mathrm{TM}_{110}$ cavity method and the associated exact solution.

\section{References}

[1] D. Polder, On the theory of ferromagnetic resonance, Phil. Mag., 40, 99-115 (Jan. 1949).

[2] A. A. Th. M. Van Trier, Guided electromagnetic waves in anisotropic media, Appl. Sci. Res., Sec. B 3, 305-371 (1953).

[3] J. O..Artman and P. E. Tannenwald, Measurement of susceptibility tensor in ferrites, J. of Applied Physics, 26, No. 9, 1124-1132 (Sept. 1955).

[4] E. G. Spencer, R. C. LeCraw, and F. Reggia, Measurement of microwave dielectric constants and tensor permeabilities of ferrite spheres, Proc. IRE 44, No. 6, 790-800 (June 1956).

[5] E. G. Spencer, L. A. Ault and R.' C. LeCraw, Intrinsic tensor permeabilities on ferrite rods, spheres and disks, Proc. IRE 44, No. 10, 1311-1317 (Oct. 1956).

[6] W. Von Aulock and J. H. Rowen, Measurement of dielectric and magnetic properties of ferromagnetic materials at microwave frequencies, Bell System Tech. J. XXXVI, No. 2 , 427-448 (Mar. 1957).

[7] I. G. MacBean, The measurement of complex permittivity and complex tensor permeability of ferrite materials at microwave frequencies, Proc. Inst. of Elec. Engrs. 104, Part B, Supplement 6, pp. 296-306 (1957).

[8] N. Ogasawara, On the measurement of permittivity and tensor permeability of thin ferrite rods, Electrotechnical Journal of Japan 3, No. 4, 126-130 (Dec. 1957).

[9] C. M. Srivastava and J. Roberts, Measurement of ferrite lossfactors at $10 \mathrm{Gc} / \mathrm{s}$, Proc. Inst. of Electrical Engineers $\mathbf{1 0 5}$, Part B, 204-209 (Mar. 1958).

[10] W. S. Carter, Apparatus for the measurement of tensor permeability and dielectric properties of ferrites at X-band frequencies, Marconi Review 22, No. 134, 154-163, Third Quarter (1959).

[11] K. H. Gothe, Experimentelle bestimmung der komponenten des permeabilitätstensors und des spektroskopischen aufspaltungsfaktors an Mg-Mn-ferriten im mikrowellengebiet, I. Annalen Der Physik 7 Folge 6, 298-306 (1960).

[12] F. Dachert and A. Schmouchkovitch, Mesure du tenseur de permeabilite des ferrites a 3000 et 8500 megacycles par seconde, J. de Physique et le Radium, Physique Appliquee, Tome 21, Supplement Au No. 3, 57A-64A (Mar. 1960).

[13] D. I. Mash and V. V. Nikol'skii, Measurement of the complex dielectric susceptibility and the magnetic susceptibility tensor of ferrites in the millimeter region, Soviet PhysicsTechnical Physics 4, No. 9, 978-980 (Mar. 1960).

[14] F. Okada, Rectangular cavities for measurement of dielectric constants and tensor permeabilities of ferrites, Defense Academy, Yakosuka, Japan, Memoirs 2, No. 1, 52-67 (Oct. 1961).

[15] C. P. Aron, The design and construction of a cavity for the measurement of the tensor susceptibility of ferrite materials, Proc. Inst. of Electrical Engineers 109, Part B, Supplement No. 23, 867-878 (1962).

[16] M. Bujatti, Measurement of tensor permeability on ferrites, Alta Frequenza XXXII, No. 5, 366-369 (1963).

[17] V. Cagan and B. Lemaire, Mesures de permittivite et de permeabilite de ferrites a differentes frequences, Electronic Magnetic Resonance and Solid Dielectrics, pp. 517-524, Proc. of the XIIth Colloque Ampere, Bordeaux, September 1963, editors: R. Servant, A. Charru, (North Holland Publ. Co.. Amsterdam, 1964).
[18] R. M. Bulley, Measurement of tensor permeability of a ferrite at $9 \mathrm{Gc} / \mathrm{s}$, Mullard Research Laboratories Report No. 533 (Apr. 1965)

[19] H. E. Bussey and L. A. Steinert, An exact solution for a cylindrical cavity containing a gyromagnetic material, Proc. of IRE 45, No. 5, 693-694 (May 1957).

[20] H. E. Bussey and L. A. Steinert, Exact solution for a gyromagnetic sample and measurements on a ferrite, IRE Trans. on Microwave Theory and Techniques MTT-6, No. 1, 72-76 (Jan. 1958).

[21] N. Ogasawara and Y. Shikata, Improved permittivity and permeability measurements, Microwaves 1, No. 5, 20-23 (Oct. 1962).

[22] N. Ogasawara and Y. Shikata, Ferromagnetic resonance study using stripline resonators, Tokyo Metropolitan University Faculty of Technology, Memoirs 12, 908-918 (1962).

[23] N. Ogasawara and S. Yamawaki, Measurement of tensor permeability on some ferrimagnetic materials at VHF band using circularly polarized resonators, International Conference on Microwaves, Circuit Theory, and Information Theory, 1964, Tokyo, Summaries of Papers, Part I Microwaves, Institute of Electrical Communication Engineers of Japan, pp. 131-132.

[24] S. P. Maxwell, A stripline cavity resonator for measuremen of magnetic and dielectric properties of ferrites at low microwave frequencies, Marconi Review 27, No. 152, 22-29, First Quarter (1964).

[25] R. A. Waldron, Theory of the stripline cavity resonator, Marconi Review 27, No. 152, 30-42, First Quarter (1964).

[26] S. P. Maxwell, A stripline cavity resonator for measurement of ferrites, Microwave Journal 9, No. 3, 99-102 (Mar. 1966).

[27] R. A. Waldron, Theory of a stripline cavity for measurement of dielectric constants and gyromagnetic resonance line widths, IEEE Trans. on Microwave Theory and Techniques MTT12, No. 1, 123-131 (Jan. 1964).

[28] R. A. Waldron and S. P. Maxwell, Note on the measurement of material properties by the stripline cavity, IEEE Trans. Microwave Theory and Techniques MTT-13, No. 5, 711, September 1965.

[29] N. Ogasawara and E. Sawado, Shape dependence of magnetic half-width of ferrimagnetic materials at lower frequency microwaves, Proc. IEEE 54, No. 4, 678-679 (Apr. 1966).

[30] R. A. Waldron, Intrinsic and external permeabilities and sus ceptibilities of gyromagnetic materials, British J. of Applied Physics 14, No. 10, 700-703, October 1963.

[31] W. E. Case, R. D. Harrington, and L. B. Schmidt, Ferrimagnetic resonance measurements using IF substitution techniques, J. Res. NBS 68C (Engr. and Instr.), No. 4, 255-259 (Oct.-Dec. 1964).

[32] ASTM Test Method C524-63T. This method is under the jurisdiction of the ASTM Committee C-25 on Ceramics for Electronics.

[33] W. E. Case, R. D. Harrington, and L. B. Schmidt, Ferrimagnetic resonance in polycrystalline ferrite and garnet disks at Lband frequencies, J. Res. NBS 68C (Engr. and Instr.), No. 2, 85-89, April-June 1964.

[34] R. M. Bozorth, Ferromagnetism, pp. 848-849 (D. Van Nostrand Company, Inc., Princeton, New Jersey, 1951). 